IJMMS 31:1 (2002) 1-9

PII. S0161171202111100

http://ijmms.hindawi.com

(c) Hindawi Publishing Corp.

\title{
TYPICALLY REAL LOGHARMONIC MAPPINGS
}

\section{ZAYID ABDULHADI}

Received 14 November 2001 and in revised form 24 January 2002

\begin{abstract}
We consider logharmonic mappings of the form $f(z)=z|z|^{2 \beta} h \bar{g}$ defined on the unit disk $U$ which are typically real. We obtain representation theorems and distortion theorems. We determine the radius of univalence and starlikeness of these mappings. Moreover, we derive a geometric characterization of such mappings.
\end{abstract}

2000 Mathematics Subject Classification: 30C55, 30C62, 49Q05.

1. Introduction. Let $H(U)$ be the linear space of all analytic functions defined in the unit disk $U=\{z=x+i y:|z|<1\}$ and let $B$ be the set of all functions $a \in H(U)$ such that $|a(z)|<1$ for all $z \in U$. A logharmonic mapping is a solution of the nonlinear elliptic partial differential equation

$$
\overline{f_{\bar{z}}}=\left(\frac{a \bar{f}}{f}\right) f_{z} ; \quad a \in B
$$

Observe that nonconstant logharmonic mappings are open and orientation preserving on $U$. If $f$ does not vanish in $U$, then $f$ is of the form

$$
f=H \bar{G},
$$

where $H$ and $G$ are in $H(U)$. On the other hand, if $f$ vanishes at zero, but has no other zeros in $U$, then $f$ admits the representation

$$
f(z)=z^{m}|z|^{2 \beta m} h(z) \overline{g(z)},
$$

where

(a) $m$ is a nonnegative integer,

(b) $\beta=\overline{a(0)}(1+a(0)) /\left(1-|a(0)|^{2}\right)$ and therefore, $\mathfrak{R} \beta>-1 / 2$,

(c) $h$ and $g$ are analytic in $U, g(0)=1$ and $h(0) \neq 0$.

In particular, a nonconstant orientation-preserving mapping $f$ is the product of an analytic function and an anti-analytic function if and only if $\beta$ is a nonnegative integer.

If $f$ is a univalent logharmonic mapping in $U$, then either $0 \notin f(U)$ and $\log f$ is univalent and harmonic on $U$, or, if $f(0)=0$, then $f$ is of the form $f(z)=z|z|^{2 \beta} h(z) \overline{g(z)}$ where $\Re \beta>-1 / 2$ and $|h(z) g(z)| \neq 0$ for $z \in U$, and where $F(\zeta)=\log f\left(e^{\zeta}\right)$ is univalent and harmonic in the half plane $\{\zeta: \mathfrak{\zeta}<0\}$. Such mappings play an important role in the theory of nonparametric minimal surfaces having a periodic Gauss map. For details see, for example, [1, 2, 3, 4, 5, 6, 7]. A logharmonic mapping $f$ is said to be 
typically real if and only if $f(z)$ is real whenever $z$ is real, and if $f$ is normalized by $f(0)=0$ and $h(0) \bar{g}(0)=1$ or equivalently by $f(0)=0$ and $h(0)=g(0)=1$. Denote by $T_{L h}$ the class of all orientation-preserving typically real logharmonic mappings. Since $f$ is orientation preserving and univalent on the interval $(-1,1)$, it follows that $m=1$ in the representation (1.3). Furthermore, if $f \in T_{L h}$, then $\beta$ (and, hence, also $a(0)$ ) has to be real and we have the relation

$$
\mathfrak{J} z \mathfrak{I} f(z)>0, \quad \forall z \in U \backslash \mathbb{R} .
$$

The class $T_{L h}$ is a compact convex set with respect to the topology of locally uniform convergence and it contains, in particular, the set $T$ of all analytic typically real functions. Our aim is to investigate the influence of the anti-analytic part of a logharmonic functions on the fundamental properties of analytic typically real functions.

In Section 2, we establish the connection between the set of typically real logharmonic mappings and the set of analytic typically real mappings (Theorem 2.1) and the set of logharmonic mappings with positive real part (Theorem 2.3). This leads us to an integral representation of $T_{L h}$. It is interesting to note that the extremal functions are univalent. As a simple application, we derive a distortion theorem for typically real logharmonic mappings for the case $a(0)=0$ (Theorem 2.6) and determine the radius of starlikeness of these mappings (Theorem 2.8). In Section 3, we consider univalent mappings in $T_{L h}$. For analytic typically real functions, it is known that if $t(z)=z+\sum_{n=2}^{\infty} a_{n} z^{n}$ is univalent in the unit disk $U$, then $t$ belongs to $T$ if and only if the image $t(U)$ is a domain symmetric with respect to the real axis.

A corresponding question for univalent typically real logharmonic functions would be as follows.

QUESTION 1.1. Let $f(z)=z|z|^{2 \beta} h(z) \overline{g(z)}$ be a univalent logharmonic mapping defined on the unit disk, and $h(0)=g(0)=1, \beta>-1 / 2$. Observe that $\beta$ (and hence $a(0))$ is real. Is it true that $f$ belongs to $T_{L h}$ if and only if the image of $f(U)$ is a symmetric domain with respect to the real axis?

The answer will be in both directions negative. We have to add additional conditions on $a(z)$ and on the image domain $\Omega=f(U)$ in order to get an affirmative answer of the question.

2. Basic properties of mappings from $T_{L h}$. We start this section with a representation theorem. We associate with each $f(z)=z|z|^{2 \beta} h(z) \overline{g(z)} \in T_{L h}$, the analytic function $\phi=z h / g \in T$.

THEOREM 2.1. (a) If $f$ is in $T_{L h}$, then $\phi \in T$.

(b) Given any $\phi \in T$ and $a \in B$ such that $\beta \in \mathbb{R}$ and hence $a(0) \in \mathbb{R}$, there are mappings $h$ and $g$ in $H(U)$ uniquely determined such that

(i) $0 \notin h g(U) ; h(0)=g(0)=1$;

(ii) $\phi=z h / g$;

(iii) the function $f(z)=z|z|^{2 \beta} h(z) \overline{g(z)} \in T_{\text {Lh }}$ is a solution of (1.1) with respect to the given $a$. 
Proof. (a) Let $f(z)=z|z|^{2 \beta} h(z) \overline{g(z)} \in T_{L h}$ be given. Then we have

$$
\mathfrak{I} \phi(z)=\mathfrak{I} \frac{z h(z)}{g(z)}=\mathfrak{I} \frac{f(z)}{|z|^{2 \beta}|g(z)|^{2}},
$$

which implies that $\phi$ is typically real.

(b) Let $\phi \in T$ and let $a \in B$ be given such that $a(0) \in \mathbb{R}$. We define

$$
\begin{aligned}
& g(z)=\exp \int_{0}^{z} \frac{s a(s) \phi^{\prime}(s)+(a(s)-1) \beta \phi(s)}{s \phi(s)(1-a(s))} d s, \\
& h(z)=\frac{\phi(z) g(z)}{z} \\
& f(z)=z|z|^{2 \beta} h(z) \overline{g(z)}=\phi(z)|z|^{2 \beta}|g(z)|^{2} .
\end{aligned}
$$

Then $h$ and $g$ are nonvanishing analytic functions defined in $U$, normalized by $h(0)=g(0)=1$ and $f$ is a solution of (1.1) with respect to the given $a$. It remains to show that $f$ is typically real. Since $\phi(z)$ is typically real and $\mathfrak{J} f(z)=|z|^{2 \beta}|g(z)|^{2} \mathfrak{J} \phi(z)$, then $f$ is typically real.

As a direct consequence we have the following corollary.

COROLlary 2.2. Let $f(z)$ be in $T_{L h}$. Then there exists a $\phi$ in $T$ such that $f$ admits the representation

$$
f(z)=z|z|^{2 \beta} h(z) \overline{g(z)}=\phi(z)|z|^{2 \beta}|g(z)|^{2},
$$

where

$$
g(z)=\exp \int_{0}^{z} \frac{s a(s) \phi^{\prime}(s)+(a(s)-1) \beta \phi(s)}{s \phi(s)(1-a(s))} d s,
$$

and $\phi=z h / g, a \in B, a(0) \in \mathbb{R}$ and hence $\beta \in \mathbb{R}$.

Now consider the subclass $T_{L h}^{\circ}$ of $T_{L h}$ that consists of all mappings $F$ from $T_{L h}$ for which $\phi=z h / g=z /\left(1-z^{2}\right)$. Then $F(z)$ is of the form

$$
F(z)=\frac{z}{1-z^{2}}|z|^{2 \beta} \exp 2 \Re \int_{0}^{z} \frac{a(s)\left(1+s^{2}\right) /\left(1-s^{2}\right)+(a(s)-1) \beta}{s(1-a(s))} d s .
$$

Denote by $P_{L h}$ the class of all logharmonic mappings $R$ defined on the unit disk $U$ which are of the form $R=H \bar{G}$, where $H$ and $G$ are in $H(U), H(0)=G(0)=1$ and such that $\Re R(z)>0$ for all $z \in U$. It contains, in particular, the set $P$ of all analytic functions with positive real part $p$ normalized by $p(0)=1$. A detailed study of this class can be found in [1].

In the next theorem we give the linkage between the class $T_{L h}$ and the class $P_{L h}$.

THEOREM 2.3. If $f(z)=z|z|^{2 \beta} h(z) \overline{g(z)} \in T_{L h}$ with respect to $a \in B, a(0) \in \mathbb{R}$ and hence $\beta \in \mathbb{R}$. Then there exists an $R \in P_{L h}$ and an $F \in T_{L h}^{\circ}$, both functions are logharmonic with respect to the same $a$ and such that

$$
f(z)=F(z) R(z)
$$


Proof. Let $f(z)=z|z|^{2 \beta} h(z) \overline{g(z)} \in T_{L h}$ with respect to a given $a \in B, a(0) \in \mathbb{R}$. Let $F \in T_{L h}^{\circ}$ with respect to the same $a$. A simple calculation implies that $f(z) / F(z)$ is a logharmonic function with respect to the same $a$. Moreover, we have

$$
\begin{aligned}
& \mathfrak{R} \frac{f(z)}{F(z)} \\
& =\mathfrak{R} \frac{\phi(z)|z|^{2 \beta} \exp 2 \mathfrak{R} \int_{0}^{z}\left(s a(s) \phi^{\prime}(s)+(a(s)-1) \beta \phi(s)\right) /(s \phi(s)(1-a(s))) d s}{\left(z /\left(1-z^{2}\right)\right)|z|^{2 \beta} \exp 2 \mathfrak{R} \int_{0}^{z}\left(a(s)\left(\left(1+s^{2}\right) /\left(1-s^{2}\right)\right)+(a(s)-1) \beta\right) /(s(1-a(s))) d s} \\
& =\frac{\exp 2 \mathfrak{R} \int_{0}^{z}\left(s a(s) \phi^{\prime}(s)+(a(s)-1) \beta \phi(s)\right) /(s \phi(s)(1-a(s))) d s}{\exp 2 \mathfrak{R} \int_{0}^{z}\left(a(s)\left(\left(1+s^{2}\right) /\left(1-s^{2}\right)\right)+(a(s)-1) \beta\right) /(s(1-a(s))) d s} \mathfrak{R} \frac{1-z^{2}}{z} \phi(z)>0 .
\end{aligned}
$$

Indeed, $\phi=z h / g \in T$ implies $\mathfrak{R}\left(\left(1-z^{2}\right) \phi(z) / z\right)>0$ (cf. [8]). Therefore, $R(z)=$ $f(z) / F(z) \in P_{\text {Lh }}$.

Our next result is a distortion theorem for the class $T_{L h}^{\circ}$ with $a(0)=0$.

LEMMA 2.4. Let $F(z)=z h(z) \overline{g(z)} \in T_{L h}^{\circ}$, then, for $z \in U$,

(i) $|F(z)| \leq|z| e^{2|z| /(1-|z|)}$;

(ii) $\left|F_{z}(z)\right| \leq\left(\left(1+|z|^{2}\right) /\left(1-|z|^{2}\right)(1-|z|)\right) e^{2|z| /(1-|z|)}$;

(iii) $\left|F_{\bar{z}}(z)\right| \leq\left(|z|\left(1+|z|^{2}\right) /\left(1-|z|^{2}\right)(1-|z|)\right) e^{2|z| /(1-|z|)}$.

Equality occurs if and only if $F(z)$ is one of the functions of the form $\bar{\eta} F_{\circ}(\eta z),|\eta|=1$, where

$$
F_{\circ}(z)=\frac{z}{1-z^{2}}\left|1-z^{2}\right| e^{\mathfrak{R}(2 z /(1-z))} .
$$

Proof. Let $F=z h(z) \overline{g(z)} \in T_{L h}^{\circ}$ with respect to a given $a \in B, a(0)=0$. Then $F$ is of the form

$$
F(z)=\frac{z}{1-z^{2}} \exp 2 \Re \int_{0}^{z} \frac{a(s)\left(1+s^{2}\right)}{s(1-a(s))\left(1-s^{2}\right)} d s .
$$

For $|z|=r$, we have

$$
\left|\frac{a(z) / z}{1-a(z)}\right| \leq \frac{1}{1-r}, \quad\left|\frac{1+z^{2}}{1-z^{2}}\right| \leq \frac{1+r^{2}}{1-r^{2}}
$$

Therefore,

$$
|F(z)| \leq \frac{r}{1-r^{2}} \exp 2 \int_{0}^{r} \frac{1+t^{2}}{(1-t)\left(1-t^{2}\right)} d t=r e^{2 r /(1-r)}
$$

Equality occurs if and only if $a(z)=\eta z,|\eta|=1$ which leads to $F(z)=\bar{\eta} F_{\circ}(\eta z)$.

The next lemma is shown in [1].

LEMMA 2.5. Let $R(z)=H(z) \overline{G(z)} \in P_{L h}$ and suppose that $a(0)=0$. Then for $z \in U$,

(i) $e^{-2|z| /(1-|z|)} \leq|R(z)| \leq e^{2|z| /(1-|z|)}$;

(ii) $\left|R_{z}(z)\right| \leq\left(2 /(1-|z|)\left(1-|z|^{2}\right)\right) e^{2|z| /(1-|z|)}$;

(iii) $\left|R_{\bar{z}}(z)\right| \leq\left(2|z| /(1-|z|)\left(1-|z|^{2}\right)\right) e^{2|z| /(1-|z|)}$. 
Equality occurs for the right-hand side inequalities if $R(z)$ is one of the functions of the form $R_{\circ}(\eta z),|\eta|=1$, where

$$
R_{\circ}(z)=\frac{1+z}{1-z}\left|\frac{1-z}{1+z}\right| e^{2|z| /(1-|z|)}
$$

and for the left-hand side inequality if $R(z)$ is one of the functions of the form

$$
\frac{1}{R_{\circ}(\eta z)}, \quad|\eta|=1
$$

Combining Lemmas 2.4 and 2.5 together with Theorem 2.3, we deduce the following distortion theorem for the class $T_{L h}$ with $a(0)=0$.

THEOREM 2.6. Let $f(z)=z h(z) \overline{g(z)} \in T_{L h}$. Then for $z \in U$,

(i) $|f(z)| \leq|z| e^{4|z| /(1-|z|)}$,

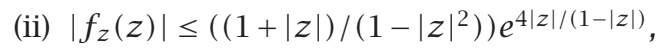

(iii) $\left|f_{\bar{z}}(z)\right| \leq\left(|z|(1+|z|) /(1-|z|)^{2}\right) e^{4|z| /(1-|z|)}$.

Equality holds for the inequalities if $f(z)$ is one of the functions of the form $\bar{\eta} f_{\circ}(\eta z)$, $|\eta|=1$, where

$$
f_{\circ}(z)=\frac{z(1-\bar{z})}{(1-z)} e^{\Re(4 z /(1-z))}
$$

REMARK 2.7. The function $f_{\circ}(z),|\eta|=1$, as it is given in (2.14), plays the role of the Koebe mapping in the set of logharmonic mappings (see [2, 5]).

In the next result we determine the radius of starlikeness for the mappings in the set $T_{L h}$.

THEOREM 2.8. Let $f(z)=z|z|^{2 \beta} h(z) \overline{g(z)} \in T_{\text {Lh }}$. Then $f$ maps the disk $\{z:|z|<$ $\left.R_{0}\right\}$, where $R_{0}=(1+\sqrt{5}-\sqrt{2+2 \sqrt{5}}) / 2$ onto a starlike domain. The upper bound is the best possible for all $a \in B$.

Proof. Let $f(z)=z|z|^{2 \beta} h(z) \overline{g(z)} \in T_{L h}$ with respect to a given $a \in B$. Then by Theorem 2.3, there exists a function $R=H \bar{G} \in P_{L h}$ and a function

$$
F(z)=\frac{z}{1-z^{2}}|z|^{2 \beta} \exp 2 \Re \int_{0}^{z} \frac{a(s)\left(\left(1+s^{2}\right)\left(1-s^{2}\right)\right)+(a(s)-1) \beta}{(s(1-a(s)))} d s \in T_{L h}^{\circ},
$$

both functions are logharmonic with respect to the given $a$, such that

$$
f(z)=F(z) R(z)
$$

From [1, Theorem 2.1], it follows that $R$ admits the representation

$$
R(z)=p(z) \exp 2 \Re \int_{0}^{z} \frac{a(s)}{1-a(s)} \frac{p^{\prime}(s)}{p(s)} d s,
$$

where $a \in B$ and $p$ is an analytic function with positive real part normalized by $p(0)=1$. 
A simple calculation leads to

$$
\begin{aligned}
\frac{\partial \arg f(z)}{\partial \theta} & =\mathfrak{R} \frac{z f_{z}(z)-\bar{z} f_{\bar{z}}(z)}{f(z)} \\
& =\mathfrak{R} \frac{z F_{z}(z)-\bar{z} F_{\bar{z}}(z)}{F(z)}+\mathfrak{R} \frac{z R_{z}(z)-\bar{z} R_{\bar{z}}(z)}{R(z)} \\
& =\mathfrak{R} \frac{1+z^{2}}{1-z^{2}}+\mathfrak{R} \frac{z p^{\prime}(z)}{p(z)}
\end{aligned}
$$

where $z=r e^{i \theta}$. Since $\mathfrak{R}\left(\left(1+z^{2}\right) /\left(1-z^{2}\right)\right) \geq\left(1-r^{2}\right) /\left(1+r^{2}\right)$ and $\mathfrak{R}\left(z p^{\prime} / p\right) \geq-2 r /(1-$ $r^{2}$ ), we obtain

$$
\mathfrak{R} \frac{z f_{z}-\bar{z} f_{\bar{z}}}{f} \geq \frac{1-r^{2}}{1+r^{2}}-\frac{2 r}{1-r^{2}}
$$

This gives

$$
\mathfrak{R} \frac{z f_{z}-\bar{z} f_{\bar{z}}}{f} \geq \frac{1-2 r-2 r^{2}-2 r^{3}+r^{4}}{\left(1+r^{2}\right)\left(1-r^{2}\right)} .
$$

Thus $\mathfrak{R}\left(\left(z f_{z}-\bar{z} f_{\bar{z}}\right) / f\right)>0$ if $1-2 r-2 r^{2}-2 r^{3}+r^{4}>0$. Therefore, the radius of starlikeness $\rho$ is the smallest positive root (less than 1) of $1-2 r-2 r^{2}-2 r^{3}+r^{4}=0$ which is $R_{0}=(1+\sqrt{5}-\sqrt{2+2 \sqrt{5}}) / 2$. We conclude that $f$ is univalent in $\{z:|z|<$ $\left.R_{0}\right\}$ and maps the circle $\left\{z:|z|<R_{0}\right\}$ onto a starlike domain. The analytic function $f(z)=z(1-z) /\left((1+z)\left(1+z^{2}\right)\right)$ belongs to the set $T$ and hence to the set $T_{L h}$ and we have $f^{\prime}\left(R_{0}\right)=0$. Hence, the upper bound $R_{0}$ is best possible for $T_{L h}$. Since $f(z)=$ $z|z|^{2 \beta} h \bar{g} \in T_{L h}$ if and only if $\phi=z h / g \in T$ (Theorem 2.1), the same bound is the best possible for all $a \in B$.

3. Univalent mappings in $T_{L h}$. Now we show that both directions of Question 1.1 do not hold in general.

Our first example shows the existence of a normalized univalent logharmonic mapping $F(z)=z H(z) \overline{G(z)}$ which belongs to $T_{L h}$ and such that $F(U)$ is not symmetric with respect to the real axis.

EXAMPLE 3.1. We define

$$
F(z)=z\left(1+\frac{i z}{8}\right)\left(1-\frac{i \bar{z}}{8}\right)=z-\frac{z y}{4}+\frac{z|z|^{2}}{64} .
$$

Then

(a) $F$ is a normalized logharmonic mapping. The normalization $F(0)=0, H(0)=$ $G(0)=1$ is obvious. It remains to show that $F$ is orientation-preserving, that is, $|a|=\left|F \overline{F_{\bar{z}}} / F_{z} \bar{F}\right|<1$ in $U$. Indeed, we have $|a(z)|=|i z /(8+2 i z)|<1 / 6$ in $U$. Observe also that the necessary condition $a(0)$ real holds since $\beta=0$;

(b) $F$ is univalent on $U$. We have for $z_{1}, z_{2}$ in $U, z_{1} \neq z_{2}$,

$$
\left|F\left(z_{1}\right)-F\left(z_{2}\right)\right|=\left|z_{1}-z_{2}\right|\left|1-\frac{\left(z_{1} y_{1}-z_{2} y_{2}\right)}{4\left(z_{1}-z_{2}\right)}+\frac{\left(z_{1}\left|z_{1}\right|^{2}-z_{2}\left|z_{2}\right|^{2}\right)}{64\left(z_{1}-z_{2}\right)}\right|>\frac{15\left|z_{1}-z_{2}\right|}{32} \text {; }
$$

(c) F is typically real. This follows directly from the fact that $F(U)$ is a pointwise stretching of $U$. We have $\arg F(z)=\arg z$; 
(d) $F(U)$ is not symmetric with respect to the real axis. A simple calculation leads to

$$
\begin{aligned}
I(t) & =\mathfrak{J} F\left(e^{i t}\right)=\sin (t)\left(1-\frac{\sin (t)}{4}+\frac{1}{64}\right), \\
I^{\prime}(t) & =\cos (t)\left(1-\frac{\sin (t)}{2}+\frac{1}{64}\right) .
\end{aligned}
$$

It follows from the equation $I^{\prime}(t)=0$ that $t= \pm \pi / 2$. We deduce for the two extrema $M=\max _{|t| \leq \pi} I(t)$ and $m=\min _{|t| \leq \pi} I(t)$ that $M=49 / 64$ and $m=-81 / 64$. Hence, $F(U)$ is not symmetric with respect to the real axis. In particular, we do not have $\overline{F(\bar{z})} \equiv F(z)$.

Our next example shows that there are univalent logharmonic mappings from $U$ onto a symmetric domain $\Omega$ which do not belong to $T_{L h}$.

EXAMPLE 3.2. Consider the function

$$
F(z)=z \frac{1+i \bar{z}}{1-i z}
$$

It follows from [4] that $F$ is a univalent logharmonic mapping from $U$ onto $U$. Hence, $F(U)$ is symmetric with respect to the real axis. But $F$ is not typically real, since the image of the interval $(-1,1)$ is the line segment which starts from $-i$, passes through the origin and ends at the point $i$. Using an appropriate approximation we can easily get univalent logharmonic mappings with the same property. For instance, we may consider a sequence of continuous functions $f_{n}^{*}\left(e^{i t}\right)=e^{i \psi_{n}(t)}, n \in \mathbb{N}$, from the unit circle $\partial U$ onto $\partial U$ such that

(i) $\psi_{n}(t)$ is a nondecreasing function of $t$ on $[0,2 \pi)$;

(ii) $\psi_{n}(2 \pi)=\psi_{n}(0)+2 \pi$;

(iii) $\int_{0}^{2 \pi} f_{n}^{*}\left(e^{i t}\right) d t=0$ and $\int_{0}^{2 \pi} e^{-i t} f_{n}^{*}\left(e^{i t}\right) d t>0$;

(iv) $\lim _{n \rightarrow \infty} f_{n}^{*}\left(e^{i t}\right)=f^{*}\left(e^{i t}\right)$ on $\partial U$.

Let $f_{n}$ be the univalent logharmonic functions with respect to the second dilatation function $a_{n}=(-i(n-1) / n) z$, that is, $f_{n}$ is a solution of (1.1) with respect to $a_{n}$. Moreover, we suppose each $f_{n}$ is a solution of the Dirichlet problem for the boundary function $f_{n}^{*}$. Then $f_{n}(0)=0,\left(f_{n}\right)_{z}(0)>0$. Since $\left\{f_{n}\right\}$ converges locally uniformly to $F$, the mappings $f_{n}$ are not typically real for $n$ large enough.

However, if the second dilatation function $a$ has real coefficients, then we have the following theorem.

THEOREM 3.3. Let $f=z|z|^{2 \beta} h(z) \overline{g(z)}$ be a univalent (orientation-preserving) logharmonic mapping defined on the unit disk $U$ and normalized by $f(0)=0, h(0)=$ $\overline{g(0)}=1$. Suppose that the second dilatation function a has real coefficients, that is, $a(z) \equiv \overline{a(\bar{z})}$. (Observe that the condition $a(0)$ real or equivalently $\beta$ real is automatically satisfied.)

(a) If $f$ is typically real, then $f(U)$ is symmetric with respect to the real axis.

(b) If $|a| \leq k<1$ on $U$ and $f(U)$ is a strictly starlike Jordan domain symmetric with respect to the real axis, then $f$ is typically real.

Proof. (a) Let $f(z)=z|z|^{2 \beta} h(z) \overline{g(z)}$ be a univalent mapping in $T_{L h}$. Then $\phi(z)=$ $z h(z) / g(z) \in T$ and has hence real coefficients. Since $a$ has real coefficients, it follows 
that $g^{\prime} / g=a /(1-a)\left(\phi^{\prime} / \phi\right)$ and $h^{\prime} / h=1 /(1-a)\left(\phi^{\prime} / \phi\right)$ have real coefficients which implies that $h(z) \equiv \overline{h(\bar{z})}, g(z) \equiv \overline{g(\bar{z})}$ and hence $f(z) \equiv \overline{f(\bar{z})}$. Therefore, $f(U)$ is symmetric with respect to the real axis.

(b) Suppose $\Omega=f(U)$ is a strictly starlike symmetric domain (i.e., every ray emitted from the origin hits $\partial f(U)$ at one point only) and let $a \in H(U)$ be a given dilatation function with real coefficients satisfying $|a(z)| \leq k<1$ on $U$. Then by [5], there is only one univalent $\operatorname{logharmonic}$ mapping from $U$ onto $\Omega$ which is a solution of (1.1) normalized by $f(0)=0$ and $h(0)>0$ and $g(0)=1$. Since $a(z)$ has real coefficients, it follows that $\overline{a(\bar{z})}=a(z)$. Moreover, $f_{1}(z)=\overline{f(\bar{z})}$ is a univalent and logharmonic mapping defined on $U$ satisfying $f_{1}(0)=0, h_{1}(0)=1>0$ and $g_{1}(0)=1$ and $f_{1}$ is a solution of the equation

$$
\overline{f_{1 \bar{z}}}=a(z) \frac{\overline{f_{1}}}{f_{1}} f_{1 z}
$$

Therefore, $f_{1} \equiv f$, that is, $f(z)=\overline{f(\bar{z})}$. Since $f$ is univalent, it follows that $f \in T_{L h}$.

It is a natural question to ask if the condition that $a$ has real coefficient is necessary for Theorem 3.3 to hold. The answer to this question is negative as the following example shows. Instead of the class $T_{L h}^{\circ}$ for which $\phi(z)=z /\left(1-z^{2}\right)$, we consider the set $T_{L h}^{+}$of univalent logharmonic (orientation-preserving) typically real mappings $f(z)=z h(z) \overline{g(z)}$ whose second dilatation function $a \in H(U),|a| \leq k<1, a(0)=0$, and whose corresponding analytic typically real function is $\phi(z)=z /\left(1+z^{2}\right)$. Relation (2.9) then becomes

$$
(z)=\frac{z}{1+z^{2}} \exp 2 \Re \int_{0}^{z} \frac{a(s)\left(1-s^{2}\right)}{s(1-a(s))\left(1+s^{2}\right)} d s,
$$

and it follows that

$$
f(z)=\frac{z}{1+z^{2}} \exp 2 \Re \int_{0}^{z} \int_{\partial U} \frac{\eta\left(1-z^{2}\right)}{(1-\eta z)\left(1+z^{2}\right)} d \mu(\eta) d z .
$$

Each one of these mappings has the property that $f \in T_{L h}$ with $f(U)=\mathbb{C} \backslash E$, where $E$ consists of two slits on the real axis containing infinity. Hence, $f(U)$ is a symmetric domain with respect to the real axis. We show that $f$ is univalent. Put $\zeta=r e^{i t}=\phi(z)=$ $z /\left(1+z^{2}\right)$ and $F(\zeta)=R(\zeta) e^{i \theta(\zeta)}$. Then $F$ is a logharmonic mapping on $D=\phi(U)$ whose second dilatation function is $A(\zeta)=a(\phi(z))$. Each radial half-line in $D$ is mapped into itself, that is, we have $\theta\left(r e^{i t}\right) \equiv t$. Moreover,

$$
\frac{r(\partial R / \partial r)}{R}=\frac{1+A}{1-A}>0
$$

holds on $D$, which implies that $F$ is univalent on $D$; and hence $f$ is a univalent function on $U$. All these properties hold independently of the coefficients of $a$, whether they are real or not. However, we cannot conclude that the relation $f(z)=\overline{f(\bar{z})}$ holds for $a$ with real coefficients. 


\section{REFERENCES}

[1] Z. Abdulhadi, Close-to-starlike logharmonic mappings, Int. J. Math. Math. Sci. 19 (1996), no. 3, 563-574.

[2] Z. Abdulhadi and D. Bshouty, Univalent functions in $H \cdot \bar{H}(D)$, Trans. Amer. Math. Soc. 305 (1988), no. 2, 841-849.

[3] Z. Abdulhadi, D. Bshouty, and W. Hengartner, Minimal surfaces whose Gauss map covers periodically the pointed upper half-sphere exactly once, preprint, 2001.

[4] Z. Abdulhadi and W. Hengartner, Spirallike logharmonic mappings, Complex Variables Theory Appl. 9 (1987), no. 2-3, 121-130.

[5] _ Univalent harmonic mappings on the left-half plane with periodic dilatations, Univalent Functions, Fractional Calculus, and Their Applications (Kōriyama, 1988), Ellis Horwood Ser. Math. Appl., Horwood, Chichester, 1989, pp. 13-28.

[6] _ Univalent logharmonic extensions onto the unit disk or onto an annulus, Current Topics in Analytic Function Theory, World Scientific Publishing, New Jersey, 1992, pp. 1-12.

[7] _ One pointed univalent logharmonic mappings, J. Math. Anal. Appl. 203 (1996), no. 2, 333-351.

[8] G. M. Goluzin, Geometric Theory of Functions of a Complex Variable, Translations of Mathematical Monographs, vol. 26, American Mathematical Society, Rhode Island, 1969.

Zayid Abdulhadi: Department of Computer Science, Mathematics and Statistics, AMERICAN UNIVERSITY OF SHARJAH, P.O. BOX 26666, SHARJAH, UNITED ARAB EMIRATES

E-mail address: zahadi@aus.ac.ae 


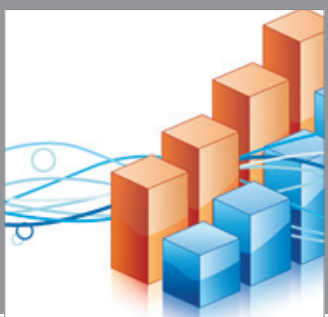

Advances in

Operations Research

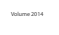

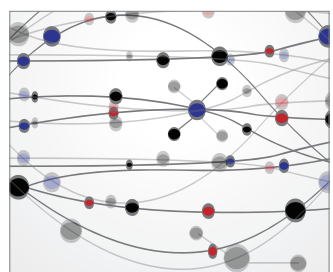

\section{The Scientific} World Journal
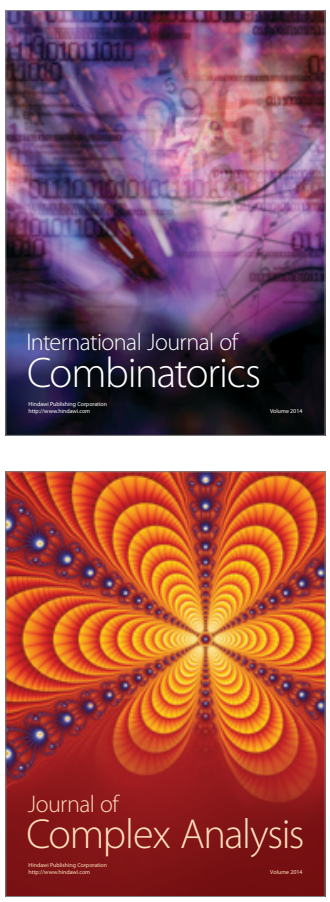

International Journal of

Mathematics and

Mathematical

Sciences
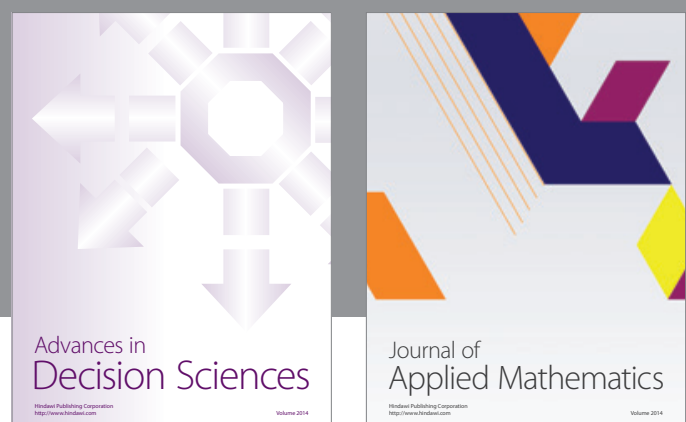

Journal of

Applied Mathematics
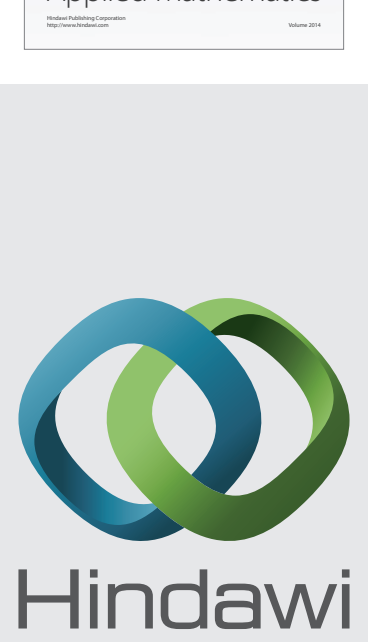

Submit your manuscripts at http://www.hindawi.com
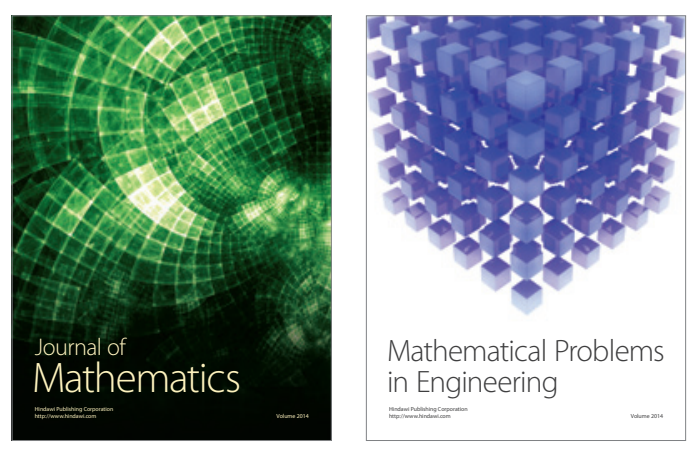

Mathematical Problems in Engineering
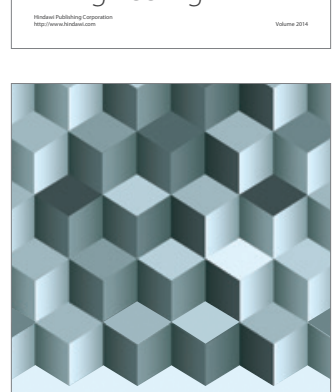

Journal of

Function Spaces
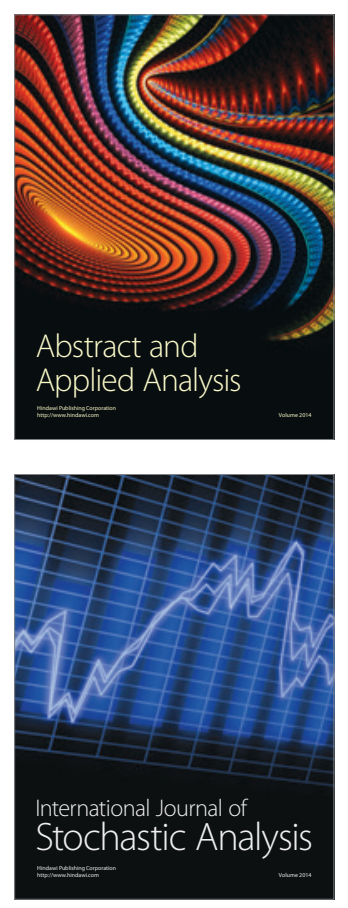

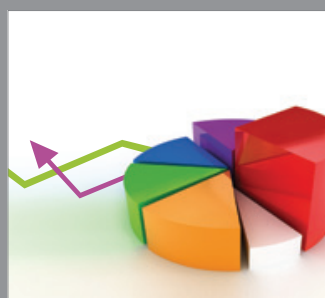

ournal of

Probability and Statistics

Promensencen
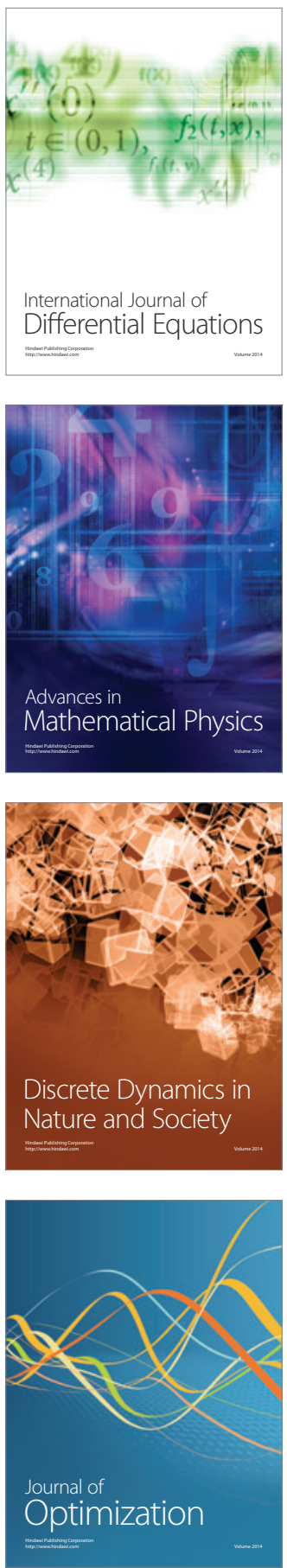